\title{
Graded Tensor Products and the Problem of Tensor Grade Computation and Reduction
}

\author{
Andrei Khrennikov \\ International Center for Mathematical Modelling \\ in Physics and Cognitive Sciences \\ Linnaeus University, Växjö, Sweden \\ Elemer E. Rosinger and Augustin van Zyl \\ Department of Mathematics, University of Pretoria \\ South-African Republic
}

July 11, 2012 


\begin{abstract}
We consider a non-negative integer valued grading function on tensor products which aims to measure the extent of entanglement. This grading, unlike most of the other measures of entanglement, is defined exclusively in terms of the tensor product. It gives a possibility to approach the notion of entanglement in a more refined manner, as the non-entangled elements are those of grade zero or one, while the rest of elements with grade at least two are entangled, and the higher its grade, the more entangled an element of the tensor product is. The problem of computing and reducing the grade is studied in products of arbitrary vector spaces over arbitrary fields.
\end{abstract}




\section{Introduction}

The notion of entanglement plays a fundamental role in modern quantum mechanics and especially quantum information theory, see,e.g., [1]. In this note we study entanglement by using a non-negative integer valued grading function defined on tensor products. This provides a possibility to bring further light upon the notoriously complex structure of entangled states. Instead of the standard dichotomy, entangled/separable, we suggest a more refined scale of degrees of entanglement, with the elements of larger grade having a higher level of entanglement. We further study the problem of the computation of the grade of a tensor, as well as the reduction of that grade. This study is done in the case of tensor products of arbitrary vector spaces over arbitrary fields, although some of the results only hold for more particular cases. This provides a possibility to apply the approach presented here, which is based on the graded tensor products, to generalizations of entanglement not only in the standard quantum mechanics over the field of complex numbers, but even to non-Archimedean quantum models, see [2] for extended review on $p$-adic physical models, see [3] on other non-Archimedean physical models, including models over nonstandard numbers, and comparing them with $p$-adic theory.

\section{Grading tensor products}

Let $E, F$ be vector spaces over the field $\mathbb{K}$. For $n \in \mathbf{N}=\{0,1,2,3, \ldots\}$, we denote by

$$
E \bigotimes^{n} F
$$

the null vector subspace of $E \otimes F$, if $n=0$, while for $n \geq 1$, we denote the set of all elements $z \in E \otimes F$ which have the form

$$
z=x_{1} \otimes y_{1}+\ldots+x_{n} \otimes y_{n}
$$

for suitable $x_{1}, \ldots, x_{n} \in E, y_{1}, \ldots, y_{n} \in F$. We call

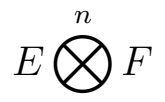


the $n$-graded subset of the tensor product $E \otimes F$. Clearly

$$
\begin{gathered}
E \bigotimes^{0} F \subseteq E \bigotimes^{1} F \subseteq \ldots \subseteq E \bigotimes^{n} F \subseteq \ldots ; \\
\left(E \bigotimes^{n} F\right) \bigcup^{n}\left(E \bigotimes^{m} F\right) \subseteq E \bigotimes^{\max \{n, m\}} F \\
\left(E \bigotimes^{n} F\right)+\left(E \bigotimes^{m} F\right) \subseteq E \bigotimes^{n+m} F, \quad n, m \in \mathbf{N} ; \\
\mathbf{K}\left(E \bigotimes^{n} F\right) \subseteq E \bigotimes^{n} F, \quad n \in \mathbb{N} ; \\
E \bigotimes_{F}=\bigcup_{n \in \mathbb{N}}\left(E \bigotimes^{n} F\right)
\end{gathered}
$$

The difficulty with these $n$-graded subsets

$$
E \bigotimes^{n} F
$$

is that, except for $n=0$, they are not closed under addition, thus they are not vector subspaces of $E \otimes F$.

Now, in view of (7), we define the tensor grading mapping

$$
g r: E \bigotimes F \longrightarrow \mathbb{N}
$$

by

$$
\operatorname{gr}(z)=\min \left\{n \in \mathbb{N} \mid z \in E \bigotimes^{n} F\right\} .
$$

and $\operatorname{gr}(z)$ is called the tensor grade of $z$.

As is well known, we have the injective mapping

$$
E \times F \ni(x, y) \longmapsto x \otimes y \in E \bigotimes F
$$

and obviously, for $z \in E \otimes F$, we have

$$
\operatorname{gr}(z) \leq 1 \Leftrightarrow z=x \otimes y \text {, for suitable } x \in E, y \in F .
$$


Let us now for $n \in \mathbb{N}$ denote

$$
E \bigotimes_{n} F=\left\{z \in E \bigotimes^{n} F \mid g r(z)=n\right\}
$$

Then clearly

$$
\begin{aligned}
& \left(E \bigotimes_{n} F\right) \bigcap\left(E \bigotimes_{m} F\right)=\phi, \quad n, m \in \mathbb{N}, n \neq m ; \\
& \left(E \bigotimes_{n} F\right) \bigcap\left(E \bigotimes^{m} F\right)=\phi, \quad n, m \in \mathbb{N}, m<n .
\end{aligned}
$$

Further we have

$$
E \bigotimes_{0} F=E \bigotimes^{0} F=\{0\}
$$

and the surjective mapping

$$
E \times F \ni(x, y) \longmapsto x \otimes y \in\{0\} \bigcup\left(E \bigotimes_{1} F\right)
$$

Consequently

$$
\mathcal{E N} \mathcal{T}(E \bigotimes F)=\bigcup_{n>1}\left(E \bigotimes_{n} F\right)
$$

is the set of entangled elements in the tensor product $E \otimes F$.

Obviously

$$
E \bigotimes F=\bigcup_{n \in \mathbb{N}}\left(E \bigotimes_{n} F\right)
$$

thus

$$
E \bigotimes F=\{0\} \bigcup\left(E \bigotimes_{1} F\right) \bigcup \mathcal{E N} \mathcal{T}(E \bigotimes F)
$$

Note 1.1. An interest in the above comes from the quanta. The state space of the composite of quantum systems is given by the tensor product of the state spaces of the component systems. Further, in case $E \otimes F$, for instance, is the state space of such a composite system, then the entangled elements $z$ in it are precisely those for which $\operatorname{gr}(z) \geq 2$, see (1.16), (1.17) above. And as is well known, the phenomenon of quantum entanglement is fundamental with respect 
to quanta. By introducing the grading function (8), (9), we obtain a measure of entanglement, according to which the higher the grade of a tensor, the more entangled it is. An important feature of the grading function gr in (8), (9) is that it is defined exclusively in terms of the respective tensor product $E \otimes F$, unlike the various measures of entanglement in the literature.

\section{Tensor Reduction}

We are interested in the following general problem of tensor reduction:

$$
\begin{aligned}
& \forall a_{1}, \ldots, a_{n}, a_{n+1} \in E, b_{1}, \ldots, b_{n}, b_{n+1} \in F: \\
& \quad\left(\begin{array}{l}
\exists c_{1}, \ldots, c_{n} \in E, d_{1}, \ldots, d_{n} \in F: \\
a_{1} \otimes b_{1}+\ldots+a_{n} \otimes b_{n}+a_{n+1} \otimes b_{n+1}=c_{1} \otimes d_{1}+\ldots+c_{n} \otimes d_{n}
\end{array}\right) \Leftrightarrow ?
\end{aligned}
$$

\subsection{Schmidt decomposition}

Theorem 3.1. Let $H_{1}, H_{2}$ be Hilbert spaces. Every representation of a tensor $u \in H_{1} \otimes H_{2}$ can be changed to a Schmidt decomposition, without increasing the number of terms in the representation. In other words, let $u=\sum_{k=1}^{n} x_{k} \otimes y_{k}$. Then there is a representation $u=$ $\sum_{k=1}^{n} \alpha_{k} u_{k} \otimes v_{k}$, with the $\left\{u_{k}\right\}$ and $\left\{v_{k}\right\}$ each being orthonormal sets, while $\alpha_{k}$ are non-negative constants.

Proof. Let $J=\operatorname{span}\left\{x_{k}\right\}_{k=1}^{n}$ and $K=\operatorname{span}\left\{y_{k}\right\}_{k=1}^{n}$. Then $J$ inherits a Hilbert space structure from $H_{1}$, and has dimension $m_{1} \leq n$. Similarly $K$ inherits the Hilbert space structure from $\mathrm{H}_{2}$, and has dimension say $m_{2} \leq n$. Observe that $u \in J \otimes K$. By the Schmidt decomposition theorem, there exists orthonormal sets $\left\{u_{k}\right\}$ in $J$, and $\left\{v_{k}\right\}$ in $K$ so that $u=\sum_{k=1}^{m} \alpha_{k} u_{k} \otimes v_{k}$, with the scalars $\left\{\alpha_{k}\right\}$ being non-negative, and $m \leq \min m_{1}, m_{2} \leq n$.

As a result, every grade-optimal representation can be changed to a Schmidt representation with the same number of terms, namely

Corollary 3.2. Let $H_{1}, H_{2}$ be Hilbert spaces and $u=\sum_{k=1}^{n} x_{k} \otimes y_{k} \in$ $H_{1} \otimes H_{2}$, with $g r(u)=n$. Then there exists a Schmidt decomposition 
$u=\sum_{k=1}^{n} \alpha_{k} u_{k} \otimes v_{k}$, with orthonormal sets $\left\{u_{k}\right\}$ and $\left\{v_{k}\right\}$, and with the scalars $\left\{\alpha_{k}\right\}$ being non-negative.

In the above theorem and corollary, the field $\mathbb{K}$ can be either $\mathbb{R}$ or $\mathbb{C}$, as is customary for Hilbert spaces.

We also note that, as is well known, the Schmidt decomposition can be effectively computed by usual methods of linear algebra, since it is closely related to the singular decomposition of matrices.

\subsection{A criterion for tensor reduction}

Let $X, Y$ be vector spaces over any field $\mathbb{K}$, and let the superscript "\#" denote linear functionals. The following proposition is essentially in [4, Proposition 1.2]. We include a proof in the Appendix.

Proposition 3.3. The following are equivalent for $u=\sum_{k=1}^{n} x_{k} \otimes y_{k} \in$ $X \otimes Y:$

(i) $u=0$;

(ii) $\sum_{k=1}^{n} \phi\left(x_{k}\right) \psi\left(y_{k}\right)=0$ for every $\phi \in X^{\#}, \psi \in Y^{\#}$.

(iii) $\sum_{k=1}^{n} \phi\left(x_{k}\right) y_{k}=0$ for every $\phi \in X^{\#}$.

(iv) $\sum_{k=1}^{n} x_{k} \psi\left(y_{k}\right)=0$ for every $\psi \in Y^{\#}$.

We use this to provide a criterion for tensor reduction.

Theorem 3.4. Let $X, Y$ be vector spaces over a field $\mathbb{K}$, and representation

$$
u=\sum_{k=1}^{n} x_{k} \otimes y_{k} \in X \bigotimes Y
$$

Then $\operatorname{gr}(u)<n$, if and only if the following criterion is satisfied: the collection $\left\{x_{k}\right\}$ or the collection $\left\{y_{k}\right\}$ are linearly dependent.

Proof. To prove the sufficiency of the criterion, suppose that the collection $\left\{x_{k}\right\}$ is linearly dependent. Then one can replace $x_{n}$ with $\sum_{k=1}^{n-1} r_{k} x_{k}$, where the $r_{k}$ 's are scalars, and use the bilinearity of " $\otimes$ " to get a representation of $u$ in terms of $n-1$ elementary tensors. Similar reasoning applies if not $\left\{x_{k}\right\}$ but $\left\{y_{k}\right\}$ is linearly dependent. 
To prove the necessity of the criterion, suppose $u$ has a representation

$$
u=\sum_{k=1}^{m} x_{k}^{\prime} \otimes y_{k}^{\prime}
$$

for some $m<n$ and $x_{k}^{\prime}$ 's and $y_{k}^{\prime}$ 's in $X, Y$ respectively, and that the criterion is not satisfied. Then, using the linear independence of the $\left\{x_{k}\right\}$, one can choose, for any integer $j, 1 \leq j \leq n$, a $\phi \in X^{\#}$ so that $\phi\left(x_{j}\right)=1$ and $\phi\left(x_{k}\right)=0$ for $k \neq j$. So, by Proposition 3.3 we get

$$
y_{j}=\sum_{k=1}^{n} \phi\left(x_{k}\right) y_{k}=\sum_{k=1}^{m} \phi\left(x_{k}^{\prime}\right) y_{k}^{\prime} .
$$

This shows that $y_{j}$ is in the span of $\left\{y_{k}^{\prime}\right\}_{k=1}^{m}$. Since $j$ is arbitrary, we get that the $y_{j}$ 's span a subspace of dimension $m$ and are therefore linearly dependent, contradicting the assumption that the criterion is not satisfied.

Although the results below can possibly be obtained by other means, it is interesting that they are also straightforward consequences of the above theorem.

Corollary 3.5. Given $u=\sum_{k=1}^{n} x_{k} \otimes y_{k} \in X \otimes Y$, then $\operatorname{gr}(u)$ is the minimum of the dimensions of $\operatorname{span}\left\{x_{k}\right\}$ and span $\left\{y_{k}\right\}$.

Corollary 3.6. If $X, Y$ have dimensions $m, n$ respectively, then for all $u \in X \otimes Y$ we have $\operatorname{gr}(u) \leq \min \{m, n\}$

A result of special interest which gives a more detailed information on the structure of the minimum number of terms in an entangled element $u \in X \otimes Y$, is in

Corollary 3.7. Given $u=\sum_{i=1}^{n} x_{i} \otimes y_{i} \in X \otimes Y$, then, after a prior permutation of the terms in this sum, and possibly of the factors $x_{i}$ with $y_{i}$, the vector $u \in X \otimes Y$ can be reduced to one of the following two forms

$$
u=\sum_{j=1}^{m} x_{j} \otimes v_{j}
$$


where $m=g r(u) \leq n$ and $v_{j}$ is in the span of $y_{1}, \ldots, y_{n}$, or alternatively, it can be reduced to

$$
u=\sum_{j=1}^{m} u_{j} \otimes y_{j}
$$

where $u_{j}$ is in the span of $x_{1}, \ldots, x_{n}$.

Proof. If $m=n$, then the proof is completed by taking $\lambda_{j}=1$.

Let therefore $m<n$. Then according to Theorem 3.4., at least one of the sets $\left\{x_{1}, \ldots, x_{n}\right\}$ or $\left\{y_{1}, \ldots, y_{n}\right\}$ is linearly dependent. Let us assume that this is the case with $\left\{x_{1}, \ldots, x_{n}\right\}$. Then in view of Corollary 3.5., and after a suitable permutation of the terms in the sum $u=\sum_{i=1}^{n} x_{i} \otimes y_{i}$, and possibly, of the factors $x_{i}$ with $y_{i}$, we can assume that the subset $\left\{x_{1}, \ldots, x_{m}\right\}$ is linearly independent and has the same span with $\left\{x_{1}, \ldots, x_{n}\right\}$. Therefore, each $x_{j}$, with $m<j \leq n$, is a linear combination of $x_{1}, \ldots, x_{m}$, namely

$$
x_{j}=\sum_{i=1}^{m} \mu_{j, i} x_{i}, \quad m<j \leq n
$$

with $\mu_{j, i} \in \mathbb{K}$. Consequently, we have

$$
\begin{aligned}
u= & \sum_{i=1}^{m} x_{i} \otimes y_{i}+\sum_{j=m+1}^{n} \sum_{i=1}^{m} \mu_{j, i} x_{i} \otimes y_{j}= \\
& =\sum_{i=1}^{m} x_{i} \otimes y_{i}+\sum_{i=1}^{m} \sum_{j=m+1}^{n} \mu_{j, i} x_{i} \otimes y_{j}= \\
& =\sum_{i=1}^{m} x_{i} \otimes\left(y_{i}+\sum_{j=m+1}^{n} \mu_{j, i} y_{j}\right)
\end{aligned}
$$

\subsection{Conclusions}

The basic result regarding the computation of the grade of an element in a tensor product is in Corollary 3.5. which is valid for arbitrary vector spaces over arbitrary fields. And that computation reduces to the computation of the dimension of two finite dimensional vector subspaces.

In the case of more particular tensor products, namely, of Hilbert spaces, which are of interest related to quanta, the computation of the grade of an element in such tensor products can also be made with 
the help of the well known and understood Schmidt decomposition.

In this way, the study of the extent of entanglement of any given element $u$ in a tensor product, study done with the help of its grade $\operatorname{gr}(u)$, is well established. Namely, if $\operatorname{gr}(u) \leq 1$, then $u$ is not entangled. On the other hand, if $\operatorname{gr}(u) \geq 2$, then the larger $\operatorname{gr}(u)$ is, the more entangled is $u$.

\section{Appendix : Proof of Proposition 3.3}

Proof. We prove $(i) \Rightarrow(i i) \Leftrightarrow(i i i) \Rightarrow(i)$.

The equivalence $(i i) \Leftrightarrow(i v)$ is similar to $(i i) \Leftrightarrow(i i i)$.

Recall that the universal mapping property of tensor products guarantees the existence, for any bilinear functional $A: X \times Y \rightarrow \mathbb{F}$, a linear mapping we denote $T_{A}: X \otimes Y \rightarrow \mathbb{F}$, that satisfies $T_{A}(x, y)=A(x, y)$ for all $(x, y) \in X \times Y$.

$(i) \Rightarrow(i i)$ : Let $\phi, \psi$ be given. Every linear mapping $T$ has the property $u=0 \Rightarrow T(u)=0$. Let $T=T_{A}$, where $A(x, y)=\phi(x) \psi(y)$.

$(i i) \Leftrightarrow(i v)$ :

$$
\begin{array}{lcl} 
& \sum_{k=1}^{n} \phi\left(x_{k}\right) \psi\left(y_{k}\right)=0 & \forall \phi \in X^{\#}, \psi \in Y^{\#} \\
\Leftrightarrow & \psi\left(\sum_{k=1}^{n} \phi\left(x_{k}\right) y_{k}=0\right) & \forall \phi \in X^{\#}, \psi \in Y^{\#} \\
\Leftrightarrow & \sum_{k} \phi\left(x_{k}\right) y_{k}=0 & \forall \phi \in X^{\#}
\end{array}
$$

(iii) $\Rightarrow(i)$ : Recall that, due to the linear structure, $T(u)=0$ for all $T: X \otimes Y \rightarrow \mathbb{F}$ implies $u=0$. And every such $T$ is the linearization of the bilinear map $A(x, y):=T(x \otimes y)$. So it is sufficient to prove that $T_{A}(u)=0$ for all bilinear $A: X \times Y \rightarrow \mathbb{F}$.

Let $A: X \times Y \rightarrow \mathbb{F}$ be given. Let $E, F$ respectively be the spans of $\left\{x_{k}\right\}$ and $\left\{y_{k}\right\}$, and $B=\left.A\right|_{E \times F}$.

Then because of the finite dimensions of $E$ and $F$ we have a representation $B(x, y)=\sum_{j=1}^{m} \alpha_{k}(x) \beta_{k}(y)$, where each $\alpha_{k}$ belongs to $E^{\#}$ and each $\beta_{k}$ belongs to $F^{\#}$. Without changing the notation, we can extend each $\alpha_{k}$ to belong to $X^{\#}$, and $\beta_{k}$ to belong to $Y^{\#}$. 
So

$$
\begin{aligned}
T_{A}(u) & =\sum_{k=1}^{n} A\left(x_{k}, y_{k}\right) \\
& =\sum_{k=1}^{n} B\left(x_{k}, y_{k}\right) \\
& =\sum_{k=1}^{n} \sum_{j=1}^{n} \alpha_{j}\left(x_{k}\right) \beta_{j}\left(y_{k}\right) \\
& =\sum_{j=1}^{n} \beta_{j}\left(\sum_{k} \alpha\left(x_{k}\right) y_{k}\right) \\
& =0,
\end{aligned}
$$

using (iii). Thus $T_{A}(u)=0$ for each $A \in B(X \times Y)$.

\section{Acknowledgments}

This research was supported by the joint project of Swedish and SouthAfrican Research Councils, "Non-Archimedean analysis: from fundamentals to applications."

\section{References}

[1] A. Zeilinger, Dance of the photons: from Einstein to quantum teleportation, Farrar, Straus and Giroux, New-York, 2010.

[2] B. Dragovich, A. Khrennikov, S. V. Kozyrev and I. V. Volovich, On p-adic mathematical physics P-Adic Numbers, Ultrametric Analysis, and Applications, 1, N 1, 1-17 (2009).

[3] E. E Rosinger, A. Khrennikov, "Beyond Archimedean SpaceTime Structure", in Advances in Quantum Theory, AIP Conf. Proc. 1327, American Institute of Physics, New York, 2011, pp. 520-526.

[4] R.A. Ryan, Introduction to Tensor Products of Banach Spaces, Springer Monographs in Mathematics,Springer-Verlag London Ltd.,2002 